\title{
Exercise Prevents Diaphragm Wasting Induced by Cigarette Smoke through Modulation of Antioxidant Genes and Metalloproteinases
}

\author{
Gracielle Vieira Ramos $\left(\mathbb{D},{ }^{1}\right.$ Alessandra Choqueta de Toledo-Arruda, ${ }^{2,3}$ \\ Clara Maria Pinheiro-Dardis, ${ }^{4}$ Camila Liyoko Suehiro, ${ }^{2}$ Thiago Luiz de Russo, ${ }^{4}$ \\ Rodolfo Paula Vieira $\mathbb{D}^{3,5,6}$ Milton Arruda Martins, ${ }^{2}$ \\ Tania Fátima Salvini, ${ }^{4}$ and João Luiz Quagliotti Durigan (D) $^{7}$
}

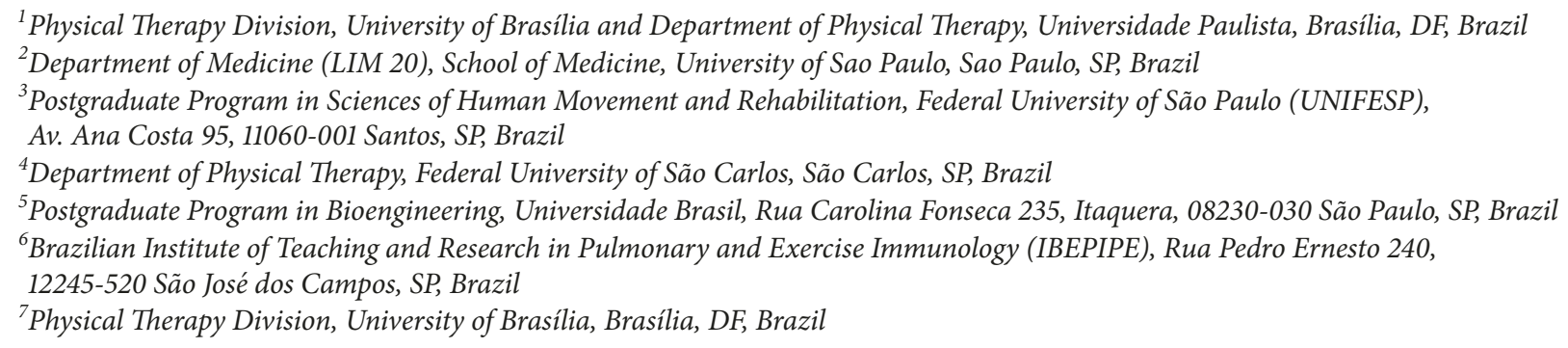

Correspondence should be addressed to Gracielle Vieira Ramos; gravr13@yahoo.com.br

Received 28 July 2017; Accepted 28 November 2017; Published 29 March 2018

Academic Editor: Ruxana Sadikot

Copyright (C) 2018 Gracielle Vieira Ramos et al. This is an open access article distributed under the Creative Commons Attribution License, which permits unrestricted use, distribution, and reproduction in any medium, provided the original work is properly cited.

\begin{abstract}
Background. The present study aimed to analyze the effects of physical training on an antioxidant canonical pathway and metalloproteinases activity in diaphragm muscle in a model of cigarette smoke-induced chronic obstructive pulmonary disease (COPD). Methods. Male mice were randomized into control, smoke, exercise, and exercise + smoke groups, which were maintained in trial period of 24 weeks. Gene expression of kelch-like ECH-associated protein 1; nuclear factor erythroid-2 like 2; and hemeoxygenasel by polymerase chain reaction was performed. Metalloproteinases 2 and 9 activities were analyzed by zymography. Exercise capacity was evaluated by treadmill exercise test before and after the protocol. Results. Aerobic training inhibited diaphragm muscle wasting induced by cigarette smoke exposure. This inhibition was associated with improved aerobic capacity in those animals that were submitted to 24 weeks of aerobic training, when compared to the control and smoke groups, which were not submitted to training. The aerobic training also downregulated the increase of matrix metalloproteinases (MMP-2 and MMP-9) and upregulated antioxidant genes, such as nuclear factor erythroid-2 like 2 (NRF2) and heme-oxygenasel (HMOX1), in exercise + smoke group compared to smoke group. Conclusions. Treadmill aerobic training protects diaphragm muscle wasting induced by cigarette smoke exposure involving upregulation of antioxidant genes and downregulation of matrix metalloproteinases.
\end{abstract}

\section{Introduction}

Chronic obstructive pulmonary disease (COPD) is associated with respiratory and peripheral muscle dysfunction. REDOX imbalance induced by cigarette smoke (CS) exposure damages lipids, proteins, and nucleic acids and is involved in diaphragm remodeling and muscle atrophy in COPD $[1,2]$. However, little is known regarding the key role of extracellular matrix (ECM) remodeling associated with oxidative stress on respiratory muscles weakness in COPD during chronic CS exposure. Metalloproteinases (MMPs), MMP-2, and MMP9 are proteolytic enzymes that play an essential role in ECM 
remodeling of skeletal muscle [3-6]. Studies have showed that MMPs also are involved in inflammation and development of interstitial fibrosis of many organs [7-9]. In addition, Yao et al. 2013 [10] have showed in mice and humans a contribution of the imbalance between tissue inhibitors of MMPs (TIMPs) and MMPs, notably between TIMP-1 and MMP-9 [10]. Increased gene expression and activity of MMP9 and MMP-2 in diaphragm muscle were also observed, which can contribute to the skeletal muscle myopathy during heart failure $[11,12]$.

It is well described that REDOX imbalance has a positive correlation with upregulation of MMPs $[13,14]$. Rajagopalan et al. (1996) [14] noticed that increased levels of free radicals, both reactive oxygen species (ROS) and reactive nitrogen species (RNS), induced activation of MMP-2 and MMP9 in cultured smooth muscle cells. Kameda et al. (2003) [13] also showed increased activity of MMP-2 and MMP9 and oxidative stress, resulting in ventricular remodeling (enlargement), followed by increased rate of morbidity and mortality of patients with coronary artery disease. Although the regulation of these enzymes is associated with oxidative stress in heart and lungs, their expression in the diaphragm after chronic CS exposure remains unknown.

Nuclear transcriptional factor (erythroid-derived 2)-like 2 (NRF2) pathway constitutes a regulatory pathway against nocive effects caused by oxidative stress through antioxidant genes upregulation $[15,16]$. Some studies have suggested that, in physiological conditions, NRF2 is maintained in the cytosol by a cluster of proteins, including its cytosolic inhibitor, kelch-like ECH-associated protein 1 (KEAP1). However, during stressful responses, redox imbalances, and cancer evolution, it dissociates from KEAP1 and translocates to the nucleus to regulate the transcription of antioxidant genes [17], such as heme-oxygenasel (HMOX1). This molecular mechanism has been observed in liver, lungs, and kidneys through KEAP1 knockdown mice approach. However in the skeletal muscle this pathway remains unclear [18]. Furthermore, a recent study has showed that NRF2 also could be associated with regulation of MMPs. In this report higher levels of MMP-9 activity in (-/-) NRF2 knockdown mice were noticed when compared to NRF2 $(+/+)$ mice after Spinal Cord Injury (SCI), suggesting that NRF2 plays a protective role in spinal cord, possibly by limiting the inflammatory response that occur after SCI via MMP-9 modulation [19]. Despite the fact that some studies have reported the critical role of NRF2 in the modulation of inflammation [19, 20], little is known about the crosstalk between MMPs and NRF2 in ECM remodeling, especially in diaphragm muscle exposed to CS $[21,22]$. Aerobic training in COPD patients alleviates symptoms, improves quality of life, and decreases exacerbation episodes and the risk of mortality in COPD. Moreover, there are evidences that aerobic training has an essential role in the prevention and progression of pulmonary disease due to cigarette smoking [23]. Interestingly, some reports showed that aerobic training also decreases the incidence of a wide range of ROS-associated diseases, including heart disease, type II diabetes, cigarette smoke-induced pulmonary disease, and certain types of cancers through the improvement of antioxidant defenses $[23,24]$. Although the literature presents a consensus about the essential role of NRF2 pathway for the protection against oxidative stress, there is no evidence about activity of NRF2 and HMOX1 pathways after aerobic training in mice exposed to cigarette smoke. In addition, there is limited information on how physical training can affect ECM turnover in respiratory muscles as well as oxidative and antioxidative response to protect skeletal muscle against CS. Therefore, the goal of this study was to analyze the effects of physical training in the NRF2-HMOX1, an antioxidant canonical pathway, and the MMP-2 and MMP-9 activities in diaphragm muscle after CS exposure. We hypothesized that chronic CS exposure would increase the MMPs activity and decrease the expression of antioxidant enzymes in diaphragm muscle, while aerobic conditioning would result in an opposite and positive effect.

\section{Material and Methods}

2.1. Animals and Experimental Design. Male C57BL/6 mice (6-8 weeks; $20.10 \pm 4.73 \mathrm{~g}$ ) were randomized into four groups of 8 animals per group: (1) control; (2) smoke (exposed to cigarette smoke); (3) exercise (submitted to treadmill training); and (4) exercise + smoke (submitted to treadmill training and exposed to cigarette smoke). The animals were housed in plastic cages under controlled environmental conditions (12-hour light/dark cycle) with free access to water and standard chow (Socil, São Paulo). The protocol of the present study was approved by the ethical committee of Faculdade de Medicina da Univesidade de São Paulo (São Paulo-Brazil) and was developed in compliance with the "Guide for care and use of laboratory animals" [25].

2.2. Cigarette Smoke Exposure Protocol. In order to induces COPD, animals were subjected to 12 commercially filtered cigarettes per day $(0,8 \mathrm{mg}$ of nicotine, $10 \mathrm{mg}$ of tar, and $10 \mathrm{mg}$ of $\mathrm{CO}$ per cigarette), corresponding to a total particulate matter concentration of $354.8 \pm 50.3 \mathrm{mg} \cdot \mathrm{m}^{-3} \cdot$ day $^{-1}$. The CS exposure was performed placing the animals into a box (inhalation chamber) maintaining controlled CO levels (250 to $350 \mathrm{ppm}$ ) for $30 \mathrm{~min}^{\mathrm{a}} \mathrm{day}^{-1}, 5$ days a week $^{-1}$ for 24 weeks. Control animals were exposed to the same protocol, but using room air [23]. In addition, carboxyhemoglobin concentration in smoke-exposed animals was kept at 10\% ( $\pm 1,3 \%)$.

\subsection{Treadmill Aerobic Training and Test. After an adaptation} to aerobic treadmill training (3 days, 15 min a day ${ }^{-1}, 25 \%$ inclination and $0.2 \mathrm{~km} / \mathrm{h}^{-1}$ ), a physical test was performed to evaluate the maximal exercise capacity (100\%), as previously described [23]. Animals were trained at 50\% of maximal exercise capacity for $60 \mathrm{~min} / \mathrm{day}, 5$ days per week for 24 weeks, and CS exposure was always performed after 1 hour of physical training. Aerobic training started on the same day as the beginning of cigarette smoke exposure and continued until the end of the experimental protocol [23].

2.4. Zymography. Diaphragm muscle was chosen because it is the most important respiratory muscle during quiet and effort breathings. Mice were weighed and diaphragm muscles 
TABLE 1: Final body weight and diaphragm muscle weight of experimental groups.

\begin{tabular}{lcccc}
\hline & Control & Smoke & Exercise & Exercise + smoke \\
\hline Initial body (g) & $38.79 \pm 3.33$ & $38.56 \pm 5.56$ & $39.51 \pm 6.39$ & $39.53 \pm 4.14$ \\
Final body (g) & $33.59 \pm 2.59^{\S}$ & $50.16 \pm 3.09^{\mathrm{a} \S}$ & $33.34 \pm 2.70^{\S}$ & $46.44 \pm 4.36^{\mathrm{a}}$ \\
Gain (\%) & 13.41 & $30.08^{\S}$ & 15.62 & $17.48^{\S}$ \\
Diaphragm weight $(\mathrm{mg})$ & $98.8 \pm 23.2$ & $60.1 \pm 13.6^{\mathrm{a}}$ & $89.5 \pm 39.4$ & $109.9 \pm 19.6^{\mathrm{b}}$ \\
\hline
\end{tabular}

Normal diaphragm muscle (control); exercise in the treadmill (exercise); group exercise in the treadmill associated to chronic cigarette smoke exposure (exercise + smoke); group submitted to chronic cigarette smoke exposure (smoke). Data are expressed as mean \pm standard deviation. $\mathrm{a}=p<0.05$ : compared to control group; $\mathrm{b}=p<0.05$ : compared to smoke group; ${ }^{\S} p<0.05$ : intragroup comparison.

were carefully removed, weighed, and frozen for zymography evaluation. Tissue extraction and zymographic analysis were performed according to current methodology [26]. Briefly, equal amounts of total protein $(30 \mu \mathrm{g} / \mathrm{lane})$, consisting of a pool of five animals per group ( $6 \mu \mathrm{g}$ per animal), were subjected to electrophoresis in triplicate. Zymography gels consisted of $10 \%$ polyacrylamide impregnated with gelatin at a final concentration of $100 \mathrm{mg} / \mathrm{ml} \mathrm{H}_{2} \mathrm{O}$ in the presence of sodium dodecyl sulfate (SDS), under nonreducing conditions. After 2 hours of electrophoresis $(100 \mathrm{~V})$, the gels were washed twice for 20 minutes in a $2.5 \%$ Triton X-100 solution and incubated at $37^{\circ} \mathrm{C}$ for $20 \mathrm{~h}$ in a substrate buffer (50 mM Tris- $\mathrm{HCl}, \mathrm{pH} 8.5,5 \mathrm{mM} \mathrm{CaCl}_{2}$, and $0.02 \% \mathrm{NaN}_{3}$ ). The gels were stained with Coomassie Brilliant Blue R-250 for 30 minutes and stained in methanol and acetic acid for 20 minutes. Gelatin-degrading enzymes were visualized as clear white bands against a blue background, indicating proteolysis of the substrate protein. The samples were also assayed in presence of $15 \mathrm{mM}$ EDTA that inhibited MMP activity. The molecular mass of gelatinolytic activities was determined by comparison to reference protein molecular mass marker, PageRulerTM Prestained Protein Ladder (Fermentas Life Sciences, Burlington, ON). Activity bands were identified following previously description, according to their molecular weights (92 kDa: pro-MMP-9; $81 \mathrm{kDa}$ : active-MMP-9; $72 \mathrm{kDa}$ : pro-MMP-2; $66 \mathrm{kDa}$ : intermediary-MMP-2; $62 \mathrm{kDa}$ : active-MMP-2) [26]. The bands found in all groups were $72-62 \mathrm{kDa}$, suggesting the activation of MMP-2 as proposed by [27]. Active bands were emphasized as described by [28]. Data is expressed as concentration of MMP-2 (i.e., the totality of integrated optical density for the MMP-2 proenzyme, intermediate, and active forms) and MMP-2 active form. Densitometry quantitative analysis of the protein bands in the zymography was performed using GeneTools v3.06 software (Syngene, Cambridge, UK).

2.5. Gene Expression by Real-Time PCR. Total RNA was isolated from diaphragm muscle using TRI-Reagent (Sigma, St. Louis, MO, USA). RNA quantity and quality were assessed using RNA Nano 6000 kit on a 2100 Bioanalyzer capillary electrophoresis system (Agilent, Santa Clara, CA, USA). Reverse transcription was carried out using High-Capacity cDNA Reverse Transcription Kit with RNase Inhibitor (Applied Biosystems). Gene expression was evaluated by quantitative real-time PCR. Two microliters of each reverse transcription product were amplified in $20 \mathrm{~L}$ of $1 \mathrm{X}$ reaction buffer (TaqMan ${ }^{\circledR}$ Universal PCR Master Mix, Applied Biosystems) using appropriate TaqMan gene expression assays in a Step One Plus Thermocycler (Applied Biosystems) for the following genes: NRF2 (Mm00477784_m1); KEAP1 (Mm00497268_ml); and HMOX1 (Mm00516005_m1). Abundance of mRNA for different genes in the diaphragm muscle was calculated with the use of $2^{-\Delta \Delta \mathrm{Ct}}$ method and Beta-actin was used as the reference gene [29].

2.6. Statistical Analysis. Kolmogorov-Smirnov and Levene tests were used to analyze the normality and homogeneity of variance. Two-way ANOVA (factors: smoke and exercise) was used to identify differences among groups. When differences were observed, Tukey test was performed. Differences were considered significant when $p<0.05$. All data were analyzed using the Statistica 7.0 software package (StatSoft Inc., Tulsa, OK, USA).

\section{Results}

3.1. Aerobic Exercise Capacity. After 24 weeks of aerobic training, the exercise time on treadmill and the maximal speed were significantly greater in those groups that trained endurance exercise, when compared to the groups that were not trained. In Figure 1, aerobic capacity improvement is noticed in the exercise group, compared to control one $(p<$ 0.05; Figure 1), as well as in exercise + smoke group compared to smoke group ( $p<0.05$; Figure 1$)$.

\subsection{Body and Muscle Weight}

3.2.1. Body Weight. Curiously, 24 weeks of cigarette smoke exposure caused an increase in body weight in both groups (smoke and smoke + exercise groups) that receive smoke tobacco when comparing their initial and final weights and when comparing them to the control group $(p<0.05)$. Moreover, body weight decrease was observed in control and exercise group, when comparing their initial and final weights $(p<0.05$; Table 1$)$.

3.2.2. Diaphragm Muscle Weight. The chronic cigarette smoke exposure reduced significantly the weight of the diaphragm muscle compared to control $(p<0.05$; Table 1$)$. However, it was also noticed that aerobic exercise prevented muscle weight loss in exercise + smoke group, when compared to the smoke group that was not trained. In the control 


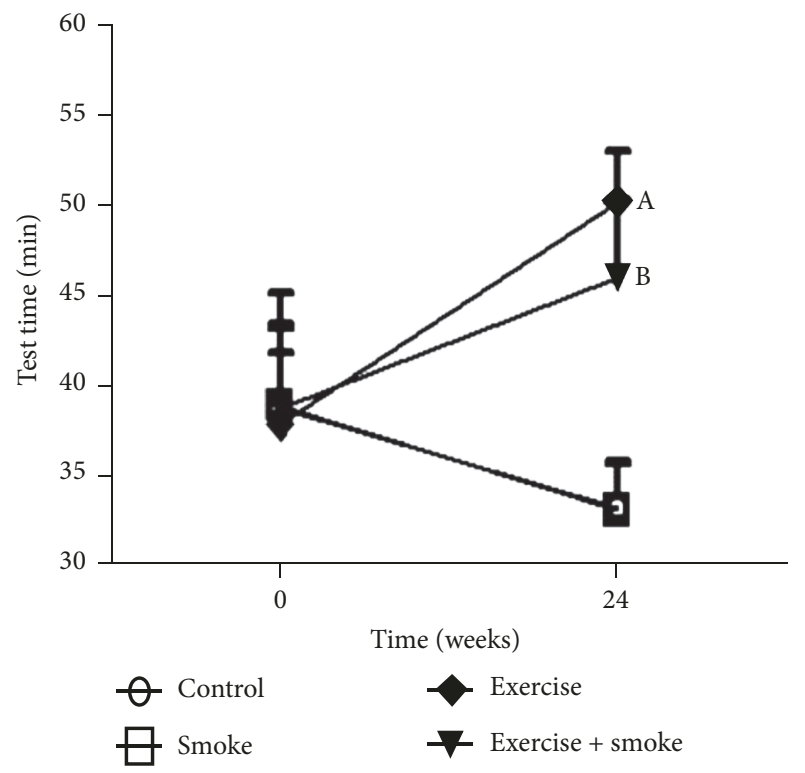

(a)

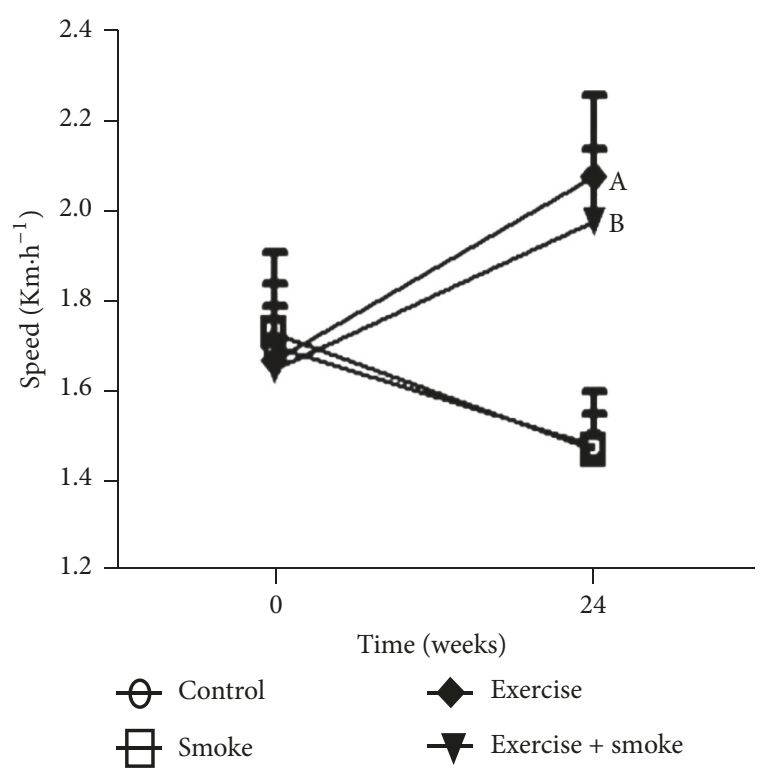

(b)

FIGURE 1: Results of the tests performed on the treadmill to evaluate the intensity of aerobic conditioning of the studied mice. Tests were performed before the beginning of the exposure to cigarette smoke (time 0 ) and 24 weeks of the protocol. (a) Time that the mice could exercise in the treadmill; (b) maximal speed reached by each mouse among the four experimental groups. Data are presented as means and standard deviation. $\mathrm{A}=p<0.05$ : compared to control group. $\mathrm{B}$ $=p<0.05$ : compared to smoke group.

and exercise groups no changes were observed after 24 weeks of training.

\subsection{Gene Expression by $q P C R$}

3.3.1. NRF2. Aerobic training, in exercise group, upregulated NFR2 gene expression in approximately four times, when compared to control and smoke groups ( $p<0.05$, resp.). There was also NRF2 gene expression increase in the exercise + smoke group, compared to the smoke and control group $(p<0.05$, resp.). Different from the expected, no differences in NRF2 gene expression between smoke and control groups were observed. However, exercise + smoke group presented increased NRF2 gene expression, when compared to exercise group $(p<0.05$, resp.) (Figure $2(a))$.

3.3.2. $H M O X$. In relation to $H M O X 1$ gene expression that is transcriptionally regulated by $N R F 2$, no difference was found between exercise and control group. On the other hand, $H M O X 1$ gene expression decrease was found in smoke group, when compared to control ( $p<0.05$, resp.). Despite HMOX1 downregulation in smoke group, aerobic training promoted increases in HMOX1 gene expression in exercise + smoke group, in comparison to control, smoke, and exercise groups, according to what could be seen in Figure 2(b). ( $p<0.05$, resp.)

3.3.3. KEAP1. Similar regulation between NRF2 and KEAP1 was noticed in qPCR. KEAP1 (NFR2 inhibitor) gene expression increase was observed, in exercise group, when compared to control and smoke group ( $p<0.05$, resp.). Similar data was observed in exercise + smoke group, when compared to other groups (control, exercise, and exercise + smoke) $(p<0.05$, resp.). An increase of KEAP1 gene expression in the exercise + smoke group compared to exercise group is also observed ( $p<0.05$, resp.). These results suggest a possible counter-regulatory mechanism between KEAP1 and NRF2 (Figure 2(c)).

3.4. MMP-2 and MMP-9 Activity of Diaphragm Muscle. The total MMP-2 activity and MMP-9 activity were elevated only in smoke group, compared to all experimental groups (control, exercise, and exercise + smoke $)(p<0.05$; Figure 3(a)). No differences were found among exercise, exercise + smoke, and control groups $(p<0.05$; Figure $3(\mathrm{~b}))$.

3.5. Discussion. Our results indicate the importance of aerobic training over ECM remodeling through MMPs and oxidative stress in diaphragm muscle mass regulation. Aerobic training maintained MMP-2 and MMP-9 activities in basal levels and increased NRF2 and HMOX1 expression (antioxidant genes), which could be associated with protective effects of physical training during chronic CS exposure. These results are of particular interest since, in humans, COPD is the most common respiratory disease, with progressive and irreversible decline of lung function, in which the diaphragm dysfunction presents an important role. We showed that mice exposure to CS for 24 weeks decreased diaphragm muscle weight $\sim 40 \%$ compared to the control group, suggesting that CS activated muscle proteolysis. We believe that muscle proteolysis observed in diaphragm muscle could be associated with inflammatory reaction and excessive ROS production. This hypothesis could be reinforced, since a decrease in HMOX1 gene expression and MMP-9 (inflammatory marker) activity increase were observed in smoke group, compared to the control group. On the other hand, the results also 


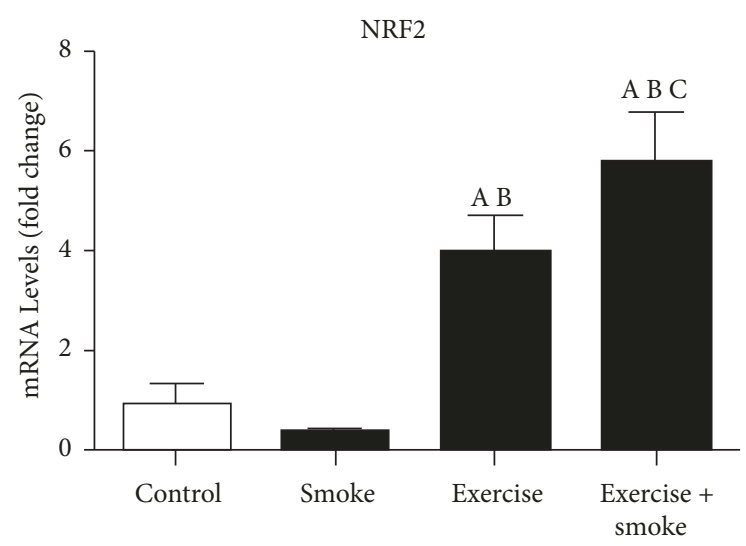

(a)

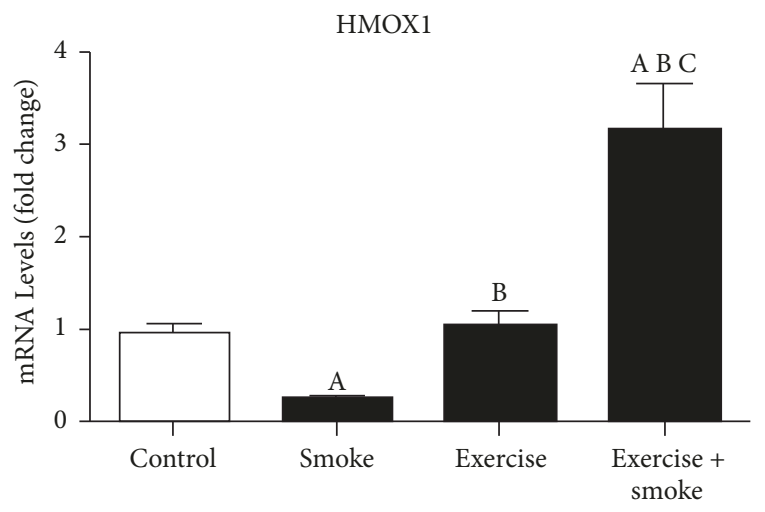

(b)

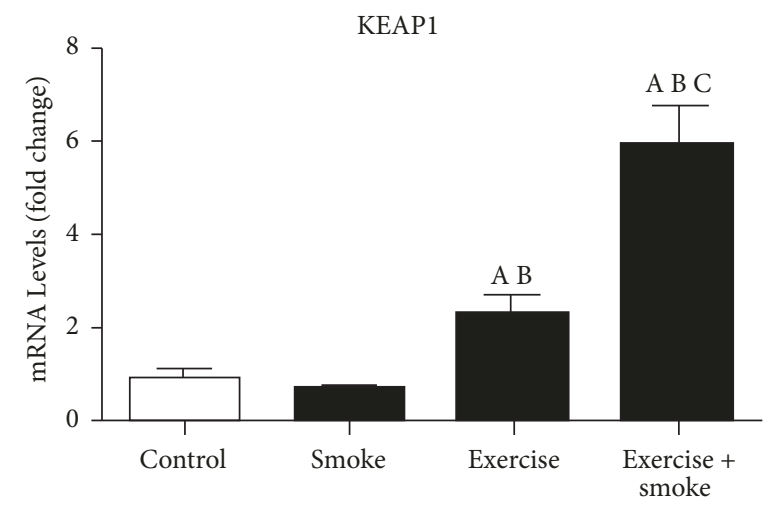

(c)

FIGURE 2: The messenger RNA (mRNA) levels of nuclear factor erythroid-2 like 2 (NRF2), heme-oxygenasel (HMOX1), and kelchlike ECH-associated protein 1 (KEAP1) of diaphragm muscle. Data are expressed as mean \pm standard deviation. $\mathrm{A}=p<0.05$ : compared to control group; $\mathrm{B}=p<0.05$ : compared to smoke group; $\mathrm{C}=$ $p<0.05$ : compared to exercise group.

showed that aerobic training is a potential intervention to prevent the imbalance between MMPs and antioxidant genes, caused by tobacco, in diaphragm muscle. Therefore, these results suggest that aerobic training has controlled muscle mass wasting through the regulation of antioxidant pathways.

Some studies have shown a regulatory mechanism between HMOX1 and transcriptional factor NRF2. Clinical

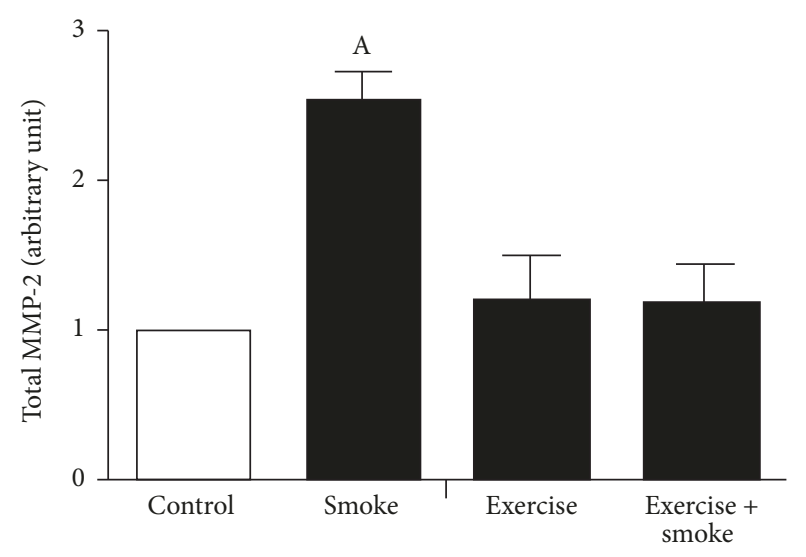

(a)

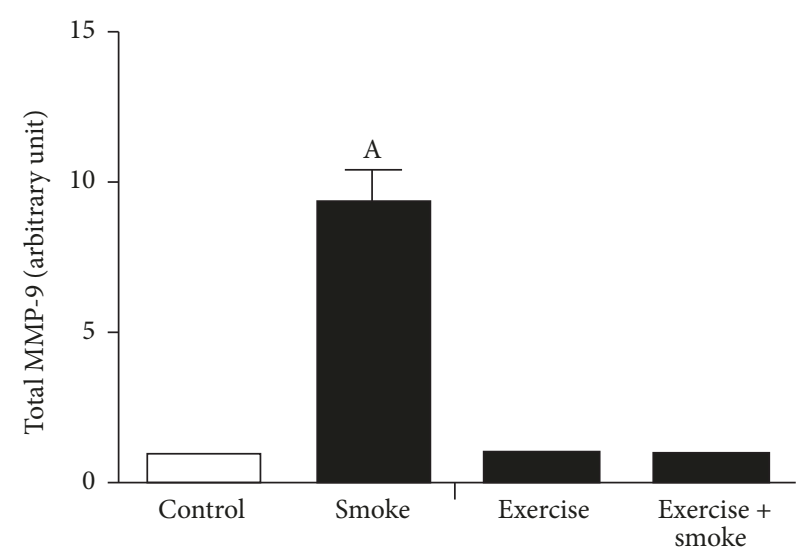

(b)

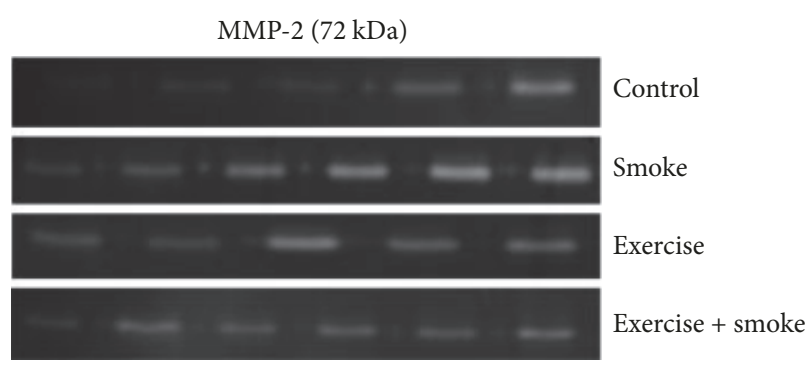

(c)

MMP-9 (92 kDa)

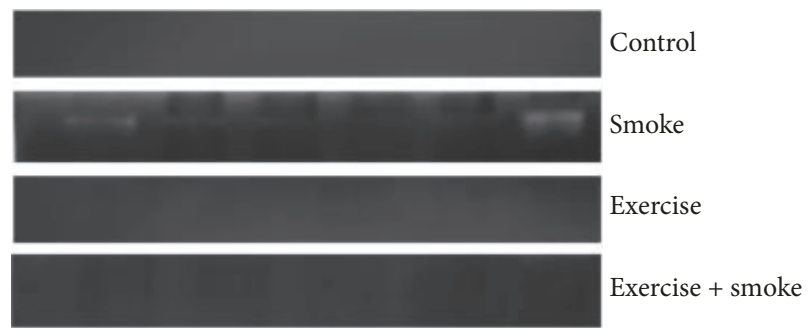

(d)

FIGURE 3: Representative gelatinolysis activity and gelatin zymographies in diafragham muscle of mice chronic cigarette smoke exposure. (a) Densitometry metalloproteinase-2 (MMP-2) gelatinolysis activity groups; (b) densitometry metalloproteinase-9 (MMP-9) gelatinolysis activity groups; (c) MMP-2 gelatin zymographies; (d) MMP-9 gelatin zymographies. $\mathrm{A}=p<0.05$ : compared to control group. 
and experimental studies have shown that NRF2 downregulation in the lungs of COPD patients and mice caused more susceptible development to emphysema after exposure to cigarette smoke [30-32]. Interestingly, in the present study, increase in NRF2 gene expression was observed in the exercise + smoke group, compared to the smoke group. It is possible to suggest that aerobic training plays a key role mediating the upregulation of the antioxidant system, in an attempt to protect muscle cells against ROS, in special, in diaphragm muscle, which was analyzed here. Curiously, we did not demonstrate difference in $H M O X 1$ gene expression between control and exercise group. Therefore, other mechanisms might be involved in the regulation between NRF2 and HMOX1 in physiological conditions [30].

It is well described that KEAP1 is a regulatory bind protein of NRF2 in the cytosol facilitating its degradation by $26 \mathrm{~S}$ proteasome $[30,31,33,34]$ Therefore, we might expect that KEAP1 would be downregulated compared to $N R F 2$, in both exercise and exercise + smoke. Different from what we expected, an increase of KEAP1 in those groups was observed. The increase of KEAP1 could be a cell mechanism to balance an excessive increase of NRF2, which is involved in the transcription of antioxidant target gene [30]. However, an increase of KEAP1 gene expression between smoke and control group was not observed. We believe that the expression of KEAP1 at basal levels might be enough to inhibit NRF2 migration into nucleus and consequently inducing antioxidants transcription, but further studies are necessary to better understand the relation between KEAP1NRF2 pathway in exercise and CS models.

The role of MMPs on ECM remodeling related to diaphragm muscle mass wasting has been poorly investigated. Our results support that CS exposure increases MMP-2 and MMP-9 activities, which might be associated with diaphragm mass muscle wasting in mice, whereas animals that were exposed to CS and submitted to exercise maintained MMPs activities similar to control group. Although the present study would not provide a direct regulatory mechanism between MMPs (MMP-2 and MMP9) and antioxidant pathway (NRF2-HMOX1), this is the first report demonstrating the protective mechanisms induced by exercise on diaphragm muscle mass decrease following CS exposure. The data reported in the present study could be reinforced by study published by Mao et al. in 2010 [19] that proved that NRF2 has a critical role in MMP-9 upregulation of mice which were knockdown to NRF2. In addition, Hindi et al. 2013 [5] showed in dystrophic muscle of mdx mice that MMP-9 inhibition improves proliferation and engraftment of myogenic cells. According to this study, the role of MMP-9 in Duchene Muscular Dystrophy could be mediated by activation of proinflammatory cytokines leading to increased inflammatory response and degradation of ECM components $[5,35]$. Future investigations will be necessary to prove the relation among NRF2, MMPs, and muscle remodeling.

It is well known that the activation of MMP-2 and MMP9 in skeletal muscle occurs despite exercise intensity and varies according to the type of muscle evaluated $[26,36]$.
Durigan and colleagues (2009) [26] observed that treadmill training at low or moderate intensities (resp., $50 \%$ or $75 \%$ of maximal speed reached in the test) during 60 minutes a day, 5 days a week for 12 weeks, did not alter MMPs activity of diaphragm muscle. These data are in agreement with the data presented here, suggesting that a training protocol at moderate intensity (50\% of maximal speed) maintained ECM remodeling in normal condition beneficiating the maintenance of diaphragm muscle remodeling in the exercise + smoke group.

In fact, training protocol at moderate intensity of exercise attenuated the harmful effects of CS exposure, regarding both MMP-9 and MMP-2 activities, in diaphragm muscle mass decrease. These results could be associated with the reduction of diaphragm muscle myopathy in CS, as previously described by Carvalho et al. (2006) [11] during heart failure. It was reported that mouse model of CS induces parenchyma mechanical dysfunction and lung remodeling, leading to impaired respiratory mechanic [23]. Probably, these mechanical changes could increase diaphragm muscle workload during training, since an increase has been observed in MMP-2 and MMP-9 activities only in CS animals. However, exercise training tends to soften the enhancement of MMPs activities, inherent to CS exposure. It is interesting to notice that, during physical exercise, abnormal dynamic in the ventilatory pattern can be observed in flow-limited patients, resulting in functional inspiratory muscle weakness by maximally shortening the muscle fibers in the diaphragm $[37,38]$. The combination of excessive mechanical loading and increased velocity of shortening of the inspiratory muscles can also predispose them to fatigue [39]. As we did not observe diaphragm ECM remodeling in a mice model of COPD in trained group, it is possible to suggest that training did not lead to abnormal dynamic ventilatory and disadvantages of diaphragm, regarding force generation.

\section{Conclusion}

Aerobic training protects diaphragm muscle wasting induced by chronic CS exposure involving upregulation of antioxidant pathway, NRF-2/HMOX, and downregulation of the matrix metalloproteinases MMP-2 and MMP-9.

\section{Conflicts of Interest}

Financial disclosure statements have been obtained, and no conflicts of interest have been reported by the authors or by any individuals in control of the content of this article.

\section{Acknowledgments}

The authors acknowledge CAPES (Coordenação de Aperfeiçoamento de Pessoal de Nível Superior) for the Postdoctorate Fellowship granted for Gracielle Vieira Ramos and they also thank Carolina Pinho for the English revision. This project was supported by Sao Paulo Research Foundation (FAPESP) Grant 2012/15165-2. 


\section{References}

[1] E. Barreiro, V. I. Peinado, J. B. Galdiz et al., "Cigarette smokeinduced oxidative stress: a role in chronic obstructive pulmonary disease skeletal muscle dysfunction," American Journal of Respiratory and Critical Care Medicine, vol. 182, no. 4, pp. 477$488,2010$.

[2] M. Doucet, A. Dubé, D. R. Joanisse et al., "Atrophy and hypertrophy signalling of the quadriceps and diaphragm in COPD," Thorax, vol. 65, no. 11, pp. 963-970, 2010.

[3] H. S. Alameddine, "Matrix metalloproteinases in skeletal muscles: friends or foes?" Neurobiology of Disease, vol. 48, no. 3, pp. 508-518, 2012.

[4] X. Chen and Y. Li, "Role of matrix metalloproteinases in skeletal muscle: migration, differentiation, regeneration and fibrosis," Cell Adhesion \& Migration, vol. 3, no. 4, pp. 337-341, 2009.

[5] S. M. Hindi, J. Shin, Y. Ogura, H. Li, and A. Kumar, "Matrix metalloproteinase-9 inhibition improves proliferation and engraftment of myogenic cells in dystrophic muscle of $\mathrm{mdx}$ mice," PLoS ONE, vol. 8, no. 8, Article ID e72121, 2013.

[6] E. Carmeli, M. Moas, A. Z. Reznick, and R. Coleman, "Matrix metalloproteinases and skeletal muscle: a brief review," Muscle \& Nerve, vol. 29, no. 2, pp. 191-197, 2004.

[7] A. Page-McCaw, A. J. Ewald, and Z. Werb, "Matrix metalloproteinases and the regulation of tissue remodelling," Nature Reviews Molecular Cell Biology, vol. 8, no. 3, pp. 221-233, 2007.

[8] T. H. Vu and Z. Werb, "Matrix metalloproteinases: effectors of development and normal physiology," Genes \& Development, vol. 14, no. 17, pp. 2123-2133, 2000.

[9] M. Giannandrea and W. C. Parks, "Diverse functions of matrix metalloproteinases during fibrosis," Disease Models \& Mechanisms, vol. 7, no. 2, pp. 193-203, 2014.

[10] H. Yao, J.-W. Hwang, I. K. Sundar et al., "SIRT1 redresses the imbalance of tissue inhibitor of matrix metalloproteinase1 and matrix metalloproteinase- 9 in the development of mouse emphysema and human COPD," American Journal of Physiology-Lung Cellular and Molecular Physiology, vol. 305, no. 9, pp. L615-L624, 2013.

[11] R. F. Carvalho, R. Dariolli, L. A. Justulin Jr. et al., "Heart failure alters matrix metalloproteinase gene expression and activity in rat skeletal muscle," International Journal of Clinical and Experimental Pathology, vol. 87, no. 6, pp. 437-443, 2006.

[12] H. M. Schiøtz Thorud, A. Stranda, J.-A. Birkeland et al., "Enhanced matrix metalloproteinase activity in skeletal muscles of rats with congestive heart failure," American Journal of Physiology-Regulatory, Integrative and Comparative Physiology, vol. 289, no. 2, pp. R389-R394, 2005.

[13] K. Kameda, T. Matsunaga, N. Abe et al., "Correlation of oxidative stress with activity of matrix metalloproteinase in patients with coronary artery disease. Possible role for left ventricular remodelling," European Heart Journal, vol. 24, no. 24, pp. 2180-2185, 2003.

[14] S. Rajagopalan, X. P. Meng, S. Ramasamy, D. G. Harrison, and Z. S. Galis, "Reactive oxygen species produced by macrophagederived foam cells regulate the activity of vascular matrix metalloproteinases in vitro: Implications for atherosclerotic plaque stability," The Journal of Clinical Investigation, vol. 98, no. 11, pp. 2572-2579, 1996.

[15] J. D. Hayes, M. McMahon, S. Chowdhry, and A. T. DinkovaKostova, "Cancer chemoprevention mechanisms mediated through the Keap1-Nrf2 pathway," Antioxidants \& Redox Signaling, vol. 13, no. 11, pp. 1713-1748, 2010.
[16] T. Nguyen, P. Nioi, and C. B. Pickett, "The Nrf2-antioxidant response element signaling pathway and its activation by oxidative stress," The Journal of Biological Chemistry, vol. 284, no. 20, pp. 13291-13295, 2009.

[17] Q. Zhong, M. Mishra, and R. A. Kowluru, “Transcription factor Nrf2-mediated antioxidant defense system in the development of diabetic retinopathy," Investigative Ophthalmology \& Visual Science, vol. 54, no. 6, pp. 3941-3948, 2013.

[18] K. Taguchi, H. Motohashi, and M. Yamamoto, "Molecular mechanisms of the Keap1-Nrf2 pathway in stress response and cancer evolution," Genes to Cells, vol. 16, no. 2, pp. 123-140, 2011.

[19] L. Mao, H. Wang, L. Qiao, and X. Wang, "Disruption of Nrf2 enhances the upregulation of nuclear factor-kappaB activity, tumor necrosis factor- $\alpha$, and matrix metalloproteinase- 9 after spinal cord injury in mice," Mediators of Inflammation, vol. 2010, Article ID 238321, 10 pages, 2010.

[20] W. Jin, H. Wang, W. Yan et al., "Disruption of Nrf2 enhances upregulation of nuclear factor- $\kappa \mathrm{B}$ activity, proinflammatory cytokines, and intercellular adhesion molecule-1 in the brain after traumatic brain injury," Mediators of Inflammation, vol. 2008, Article ID 725174, 7 pages, 2008.

[21] C. Jácome and A. Marques, "Pulmonary rehabilitation for mild COPD: A systematic review," Respiratory Care, vol. 59, no. 4, pp. 588-594, 2014.

[22] A. Singh, G. Ling, A. N. Suhasini et al., "Nrf2-dependent sulfiredoxin-1 expression protects against cigarette smokeinduced oxidative stress in lungs," Free Radical Biology \& Medicine, vol. 46, no. 3, pp. 376-386, 2009.

[23] A. C. Toledo, R. M. Magalhaes, D. C. Hizume et al., "Aerobic exercise attenuates pulmonary injury induced by exposure to cigarette smoke," European Respiratory Journal, vol. 39, no. 2, pp. 254-264, 2012.

[24] Z. Radak, H. Y. Chung, and S. Goto, "Systemic adaptation to oxidative challenge induced by regular exercise," Free Radical Biology \& Medicine, vol. 44, no. 2, pp. 153-159, 2008.

[25] Council NR, Guide for the Care and Use of Laboratory Animals, National Academies Press, Wash, USA, 8th edition, 2011.

[26] J. L. Q. Durigan, S. M. Peviani, T. L. Russo et al., "Physical training leads to remodeling of diaphragm muscle in asthma model," International Journal of Sports Medicine, vol. 30, no. 6, pp. 430-434, 2009.

[27] W. M. Frederiks and O. R. F. Mook, "Metabolic mapping of proteinase activity with emphasis on in situ zymography of gelatinases: Review and protocols," Journal of Histochemistry \& Cytochemistry, vol. 52, no. 6, pp. 711-722, 2004.

[28] R. C. Marqueti, J. Prestes, M. Paschoal et al., "Matrix metallopeptidase 2 activity in tendon regions: Effects of mechanical loading exercise associated to anabolic-androgenic steroids," European Journal of Applied Physiology, vol. 104, no. 6, pp. 10871093, 2008.

[29] K. J. Livak and T. D. Schmittgen, "Analysis of Relative Gene Expression Data Using Real-Time Quantitative PCR and the $2^{-\triangle \Delta C T}$ Method," Methods, vol. 25, no. 4, pp. 402-408, 2001.

[30] D. Goven, A. Boutten, V. Leçon-Malas, J. Boczkowski, and M. Bonay, "Prolonged cigarette smoke exposure decreases heme oxygenase-1 and alters Nrf2 and Bach1 expression in human macrophages: Roles of the MAP kinases ERK1/2 and JNK," FEBS Letters, vol. 583, no. 21, pp. 3508-3518, 2009.

[31] T. Iizuka, Y. Ishii, K. Itoh et al., "Nrf2-deficient mice are highly susceptible to cigarette smoke-induced emphysema," Genes to Cells, vol. 10, no. 12, pp. 1113-1125, 2005. 
[32] T. Rangasamy, C. Y. Cho, R. K. Thimmulappa et al., "Genetic ablation of Nrf2 enhances susceptibility to cigarette smokeinduced emphysema in mice," The Journal of Clinical Investigation, vol. 114, no. 9, pp. 1248-1259, 2004.

[33] D. J. Blake, A. Singh, P. Kombairaju et al., "Deletion of Keap1 in the lung attenuates acute cigarette smoke-induced oxidative stress and inflammation," American Journal of Respiratory Cell and Molecular Biology, vol. 42, no. 5, pp. 524-536, 2010.

[34] T. W. Kensler, N. Wakabayashi, and S. Biswal, "Cell survival responses to environmental stresses via the Keap1-Nrf2-ARE pathway," Annual Review of Pharmacology and Toxicology, vol. 47, pp. 89-116, 2007.

[35] S. B. P. Chargé and M. A. Rudnicki, "Cellular and molecular regulation of muscle regeneration," Physiological Reviews, vol. 84, no. 1, pp. 209-238, 2004.

[36] E. Carmeli, M. Moas, S. Lennon, and S. K. Powers, "High intensity exercise increases expression of matrix metalloproteinases in fast skeletal muscle fibres," Experimental Physiology, vol. 90, no. 4, pp. 613-619, 2005.

[37] E. Paulin, W. P. S. Yamaguti, M. C. Chammas et al., "Influence of diaphragmatic mobility on exercise tolerance and dyspnea in patients with COPD," Respiratory Medicine, vol. 101, no. 10, pp. 2113-2118, 2007.

[38] C. Sinderby, J. Spahija, J. Beck et al., "Diaphragm activation during exercise in chronic obstructive pulmonary disease," American Journal of Respiratory and Critical Care Medicine, vol. 163, no. 7, pp. 1637-1641, 2001.

[39] D. E. O'Donnell and P. Laveneziana, "Dyspnea and activity limitation in COPD: Mechanical factors," COPD-Journal of Chronic Obstructive Pulmonary Disease, vol. 4, no. 3, pp. 225236, 2007. 


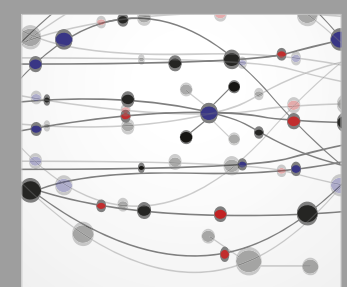

The Scientific World Journal
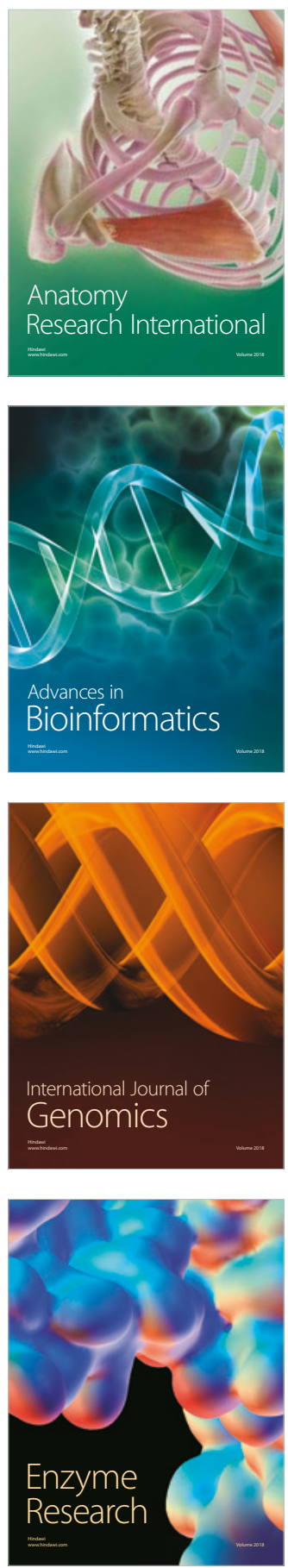
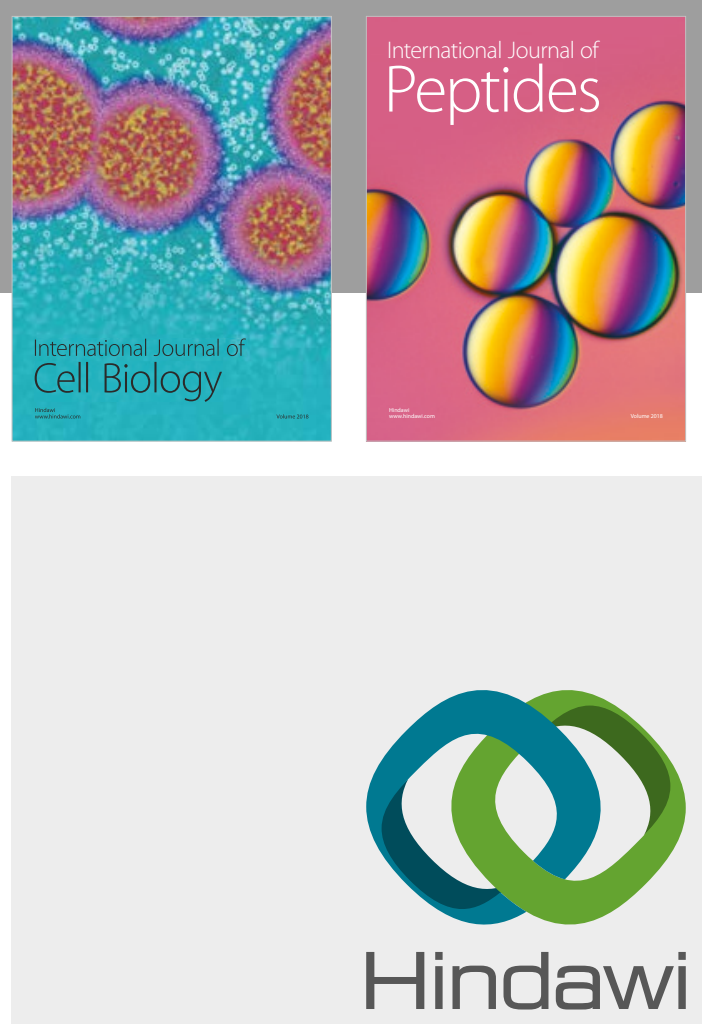

Submit your manuscripts at

www.hindawi.com
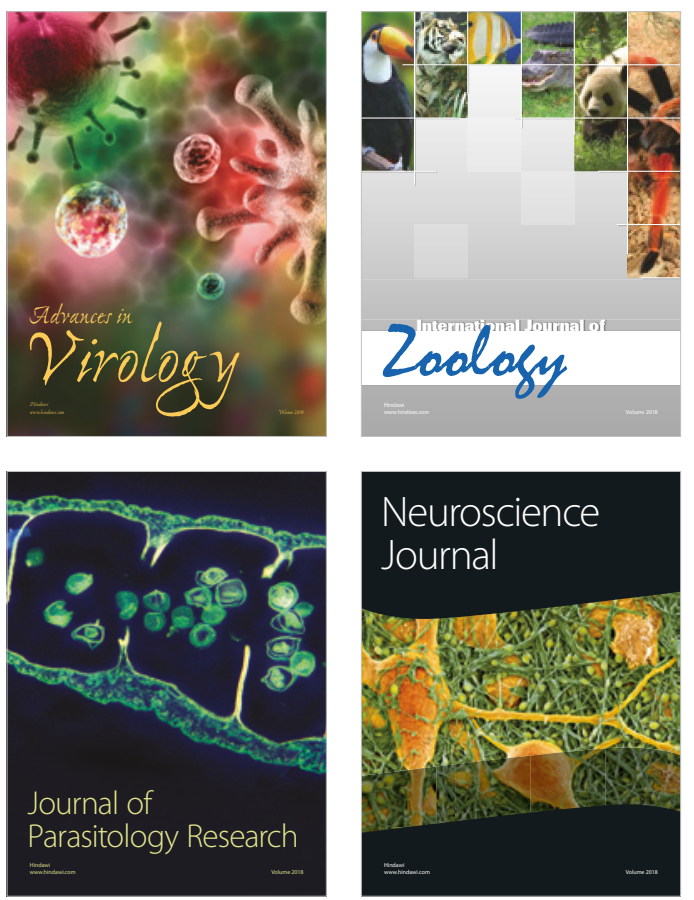
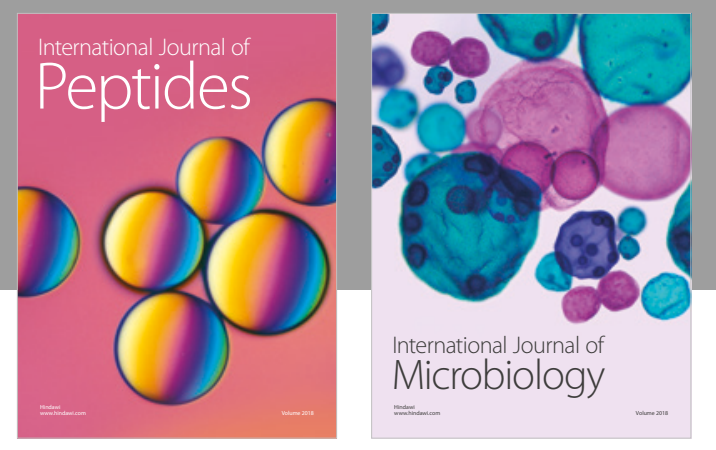

nternational Journal of Microbiology
Journal of
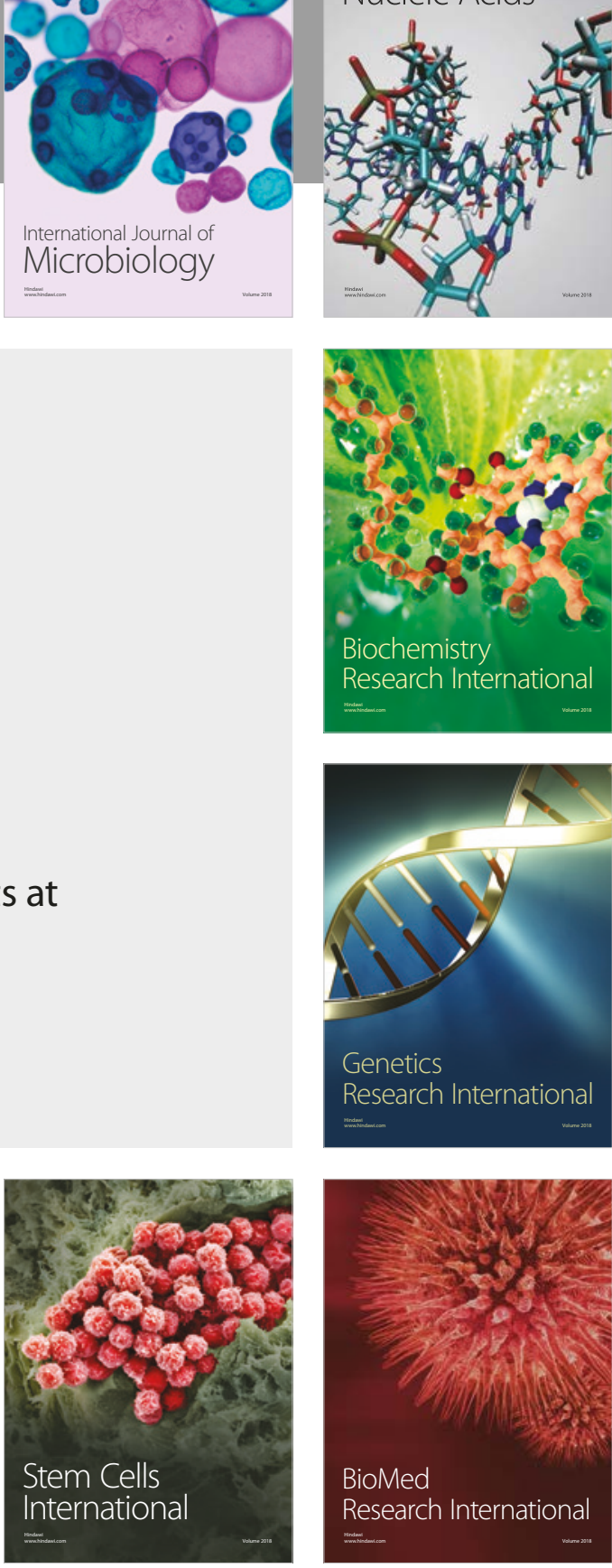
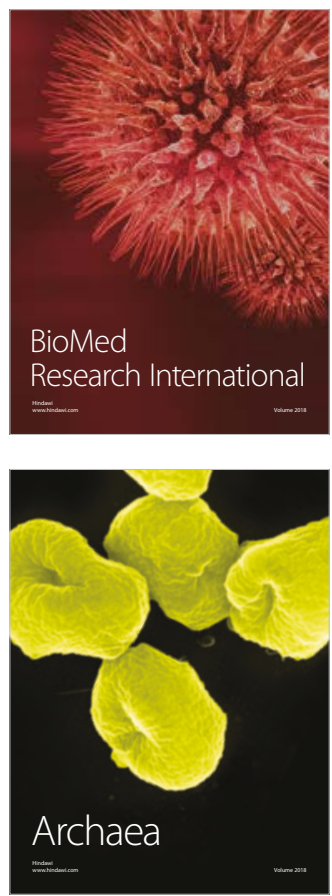